\title{
APPLICATION OF EXPLICITLY CORRELATED GAUSSIAN FUNCTIONS TO LARGE SCALE CALCULATIONS ON SMALL ATOMS AND MOLECULES
}

\author{
Jacek Komasa ${ }^{1}$, Wojciech Cencek ${ }^{1}$, and Jacek Rychlewski ${ }^{1,2}$ \\ 'Department of Chemistry, Adam Mickiewicz University, Grunwaldzka 6, \\ 60-780 Poznań, Poland \\ ${ }^{2}$ Poznań Supercomputing and Networking Center, Wieniawskiego 17/19, \\ 61-712 Poznań, Poland \\ e-mail: komasa@rose.man.poznan.pl, cencek@rose.man.poznan.pl, \\ rychlew@rose.man.poznan.pl
}

\section{Abstract}

Exponentially Correlated Gaussian wave functions are applied to variational calculations of the total electronic energy of several a few-electron atomic and molecular systems. It is shown that this powerful approach enables to obtain extremely accurate results not only for two-electron systems but also for three- and four-electron atoms and molecules.

\section{Introduction}

One of the major tasks of the computational quantum chemistry is to search for solutions of the Schrodinger equation for atomic and molecular systems. The solution presents a wave function, $\Psi$, potentially bearing complete description of the system, and its total energy, E. Unfortunately, the Schrödinger equation is very complicated-it is composed of various singular and differential operators and, moreover, contains generally 3 times more variables than particles composing the system. Therefore, in practice, we are not able to find exact analytical solutions, except for trivial one-electron cases. It is natural then looking for simplifications either in the Schrödinger equation itself or in the solving process. This, however, causes that practically every solution of the Schrödinger equation is contaminated by an inherent error. The main goal of the quantum chemistry, since the first days of its existence, is to develop methods reducing this error as much as possible. Since 1990 our research has aimed at finding very accurate solutions of the Schrödinger equation for small a few-electron atoms and molecules.

The quality of the wave function can be assessed through the energy it yields - the observable accessible experimentally. The wave function enables also theoretical determination of many other observables which can be compared with 
measurable quantities. It supplies an additional source of information about the quality of the wave function. Still, the energy remains the main criterion of the quality.

The usual objective of interest is an energy difference, i.e. transition, binding, interaction energy. However, also an absolute energy, especially when known with high accuracy, plays important role in quantum chemistry:

- it allows to investigate more subtle energetic effects, i.e. relativistic or radiative phenomena, and hence, to penetrate deeper and deeper into the nature of the matter;

- serves as a benchmark for new computational methods;

- enables correct interpretation of experimental data;

- supports determination of bulk properties.

There are two basic approaches to the total energy calculations:

- the traditional method-based on the one-electron approximation-the Hartree-Fock procedure (HF), optionally followed by the Configuration Interaction method $(\mathrm{CI})$;

- the explicitly correlated wave function method-a trial wave function contains explicitly an interelectron distance variable (e.g. Kołos-Wolniewicz (KW) wave function), so far applied successfully only to 2-electron systems and 3-electron atoms.

For many small systems it is possible to determine an accurate total energy experimentally, setting this way a reference point to the assessment of the energy error, $\Delta E$, made in particular computation. The following diagram (Fig. 1) presents the dependence of the error in microhartree, $\mu E_{H}\left(1 E_{H}=4.3597482 \cdot\right.$ $\left.10^{-18} \mathrm{~J}\right)$, made with the methods mentioned above, on the size of the system expressed by the number of electrons.

\section{Method}

A system under consideration is defined by a Hamiltonian, $\hat{H}-$ a quantum-mechanical operator describing the kinetics and all the interactions which shall be taken into account in the model. The general nonrelativistic Hamiltonian of an $n$-electron $N$-nucleus molecule can be written down as:

$$
\begin{aligned}
\hat{H} & =-\frac{\hbar^{2}}{2 m} \sum_{i=1}^{n} \nabla_{i}^{2}-\sum_{I=1}^{N} \frac{\hbar^{2}}{2 M_{I}} \nabla_{I}^{2}+\sum_{i=1}^{n-1} \sum_{j=i+1}^{n} \frac{e^{2}}{4 \pi \varepsilon_{0}} \frac{1}{\left|\vec{r}_{i}-\vec{r}_{j}\right|} \\
& +\sum_{I=1}^{N-1} \sum_{J=I+1}^{N} \frac{e^{2}}{4 \pi \varepsilon_{0}} \frac{Z_{I} Z_{J}}{\left|\vec{R}_{I}-\vec{R}_{J}\right|}-\sum_{I=1}^{N} \sum_{i=1}^{n} \frac{e^{2}}{4 \pi \varepsilon_{0}} \frac{Z_{I}}{\left|\vec{r}_{i}-\vec{R}_{I}\right|},
\end{aligned}
$$




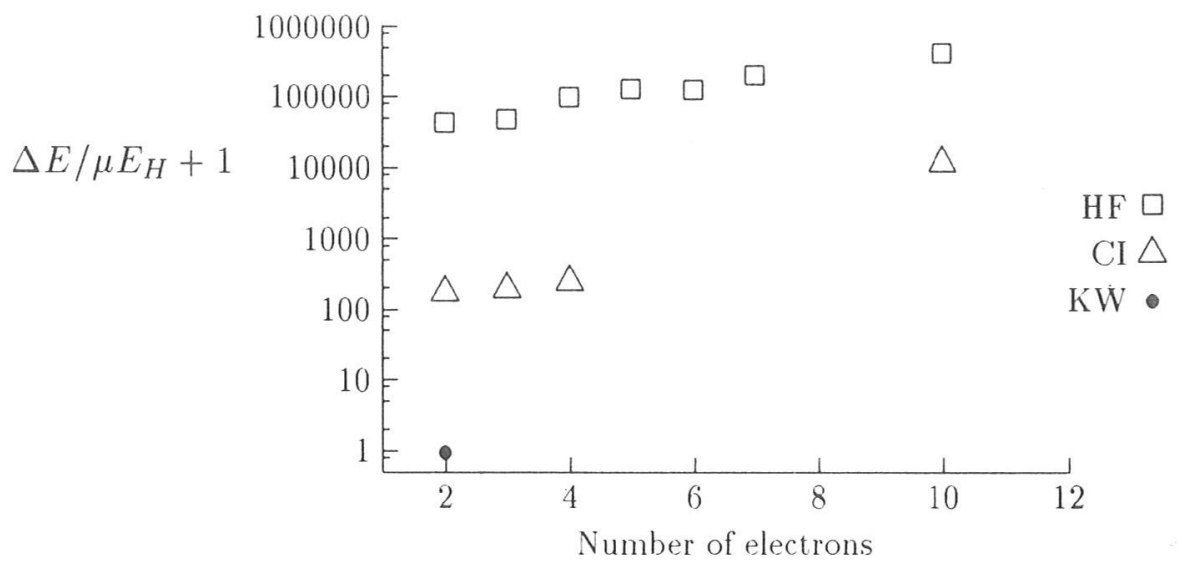

Figure 1: Total energy error, $\Delta E$, obtained from $\operatorname{HF}(\cdot)$ and $\mathrm{CI}(\Delta)$ calculations and from explicitly correlated calculations with Kołos-Wolniewicz wave function $(\mathrm{KW}, \cdot)$. Notice a lack of high accuracy results for systems with more than two electrons.

where $\vec{r}_{i}, \vec{R}_{I}$ are 3 -dimensional vectors pointing at i-th electron and I-th nucleus, respectively, $m$ and $\mathrm{e}$ are the mass and the charge of the electron, $M_{I}$ and $Z_{I}-$ the mass and the charge of the I-th nucleus. $\hbar, \varepsilon_{0}$ and $\pi$ are constants with their usual meaning. As the electrons are thousands times lighter and move much faster than nuclei, the second term in the Hamiltonian (1) is usually dropped. It corresponds to a clamped nuclei model which considers molecule as a rigid skeleton of nuclei surrounded by moving electrons. The variational method applied to solve the Schrödinger equation

$$
\hat{H} \Psi=E \Psi
$$

has an important feature: always yields energy, $E$, greater or at most equal to the exact value, $E_{0}$ :

$$
E \geq E_{0}
$$

It allows to search for the wave function $\Psi$ using the criterion of generating as low energy as possible. The variational principle (3) supplies a scale for assessing the quality of trial wave functions.

To obtain the wave function in an analytical form we will apply the algebraic approximation: $\Psi$ is represented as a linear combination of a finite number of known basis functions. $\psi_{k}$ :

$$
\Psi=\sum_{k=1}^{K} c_{k} \psi_{k} .
$$

The knowledge of the basis functions enables to look for the algebraic expansion 
coefficients instead of a general wave function of a completely unknown shape. In other words, we replace the lack of knowledge of $\Psi$ by the lack of knowledge of the set of the coefficients $c_{k}$.

\section{Basis functions}

At this point the choice of the analytical form of the basis functions, $\psi_{k}$, becomes a basic matter. A significant contribution in this field comes from two Polish scientists. Włodzimierz Kołos and Lutosław Wolniewicz applied the basis functions in the form of two-electron James-Coolidge (JC) [1] function written in elliptical coordinates as

$$
\psi_{k}\left(\lambda_{1}, \lambda_{2}, \mu_{1}, \mu_{2}, r_{12}\right)=\exp \left[-\delta\left(\lambda_{1}+\lambda_{2}\right)\right] \lambda_{1}^{m} \mu_{1}^{n} \lambda_{2}^{j} \mu_{2}^{i}\left(2 r_{12} / R\right)^{p},
$$

where $r_{12}$ and $R$ describe interelectron and internuclear distances, respectively. $\delta$ is a real and $m, n, j, i$ and $p$ are integer variational parameters. This function belongs to the class of the explicitly correlated functions. In 1964 Kołos and Wolniewicz [2, 3], using the generalized form of the JC wave function, computed a variational energy of molecular hydrogen. Their value was lower than the experimental one, apparently breaking the variational rule. Correctness of their calculations and even validity of the Schrödinger equation was questioned until few years later the experiment was revised-it turned out that the computations were correct. It was a significant success of theory in connection with the computational technique. Today the Kołos-Wolniewicz wave function allows to solve the Schrödinger equation for two-electron two-nucleus systems with practically arbitrary accuracy. For the last 30 years it has been serving as a reference point for other wave functions and methods in this area of application. Unfortunately, it has never been generalized to larger systems. Undoubtedly, the results by Kołos and Wolniewicz promoted the quantum chemistry to the level of the quantitative method able to answer detailed questions concerning two-electron molecules, and hence, the chemical bonding.

Another, the most common way of treating the electron correlation is an application of the CI wave function. It is equivalent to regarding $\psi_{k}$ of Eq. (4) in the form of determinants built of one-electron molecular functions (orbitals). Theoretically such an expansion converges to the exact wave function. In practice, however, astronomical number of terms including functions with very high angular momentum would be required to reach satisfactory accuracy. The CI wave function, in contrast to the KW wave function, does not contain explicitly the interelectron distance variable, $r_{i j}$, which is the main reason for the very slow convergence. For a few-electron systems this method yields accuracy, $\Delta \mathrm{E}$, of tens and hundreds of $\mathrm{cm}^{-1}$ (see Table 1). 
Table 1: Total electronic energy error for chosen a few-electron systems obtained from the best CI wave functions available in the literature.

\begin{tabular}{lccc}
\hline $\begin{array}{l}\text { System } \\
\left(1 \text { bohr }=0.529177249 \cdot 10^{-10} \mathrm{~m}\right)\end{array}$ & $\begin{array}{c}\text { Number } \\
\text { of electrons }\end{array}$ & $\begin{array}{c}\Delta E \\
\mathrm{~cm}^{-1}\end{array}$ & Reference \\
\hline $\mathrm{H}_{2}(\mathrm{R}=1.4011$ bohr $)$ & 2 & 38 & {$[4]$} \\
$\mathrm{He}_{2}^{+}(\mathrm{R}=2.043$ bohr $)$ & 3 & 78 & {$[5]$} \\
$\mathrm{H}_{3}\left(\mathrm{R}_{1}=\mathrm{R}_{2}=1.757\right.$ bohr $)$ & 3 & 43 & {$[6]$} \\
$\mathrm{Li}$ & 3 & 63 & {$[7]$} \\
$\mathrm{Be}$ & 4 & 54 & {$[8]$} \\
$\mathrm{He}(\mathrm{R}=5.6$ bohr $)$ & 4 & 301 & {$[9]$} \\
$\mathrm{LiH}(\mathrm{R}=3.015$ bohr $)$ & 4 & 318 & {$[10]$} \\
\hline
\end{tabular}

In 1989 Enrico Clementi following the example of Hylleraas [11], created an explicitly correlated version of the CI wave function (H-CI). He introduced the correlation factor replacing the basis function $\psi_{k}$ by $\left(1+\mathrm{r}_{i j}\right) \psi_{k}$. Indeed, compared to the classic CI the convergence has improved but this approach has turned out computationally so inconvenient that in 1991 Clementi estimated time required to obtain accuracy of about $10 \mathrm{~cm}^{-1}$ for the 3-electron $\mathrm{H}_{3}$ molecule as 3500 years of computation on an IBM 3090 class machine. He predicted that, with some optimistic assumptions, this time could be reduced to 10-20 years [7, 12].

At this moment of development, the quantum chemistry offered a possibility of performing either very accurate calculations limited to at most two-electron systems or calculations on larger systems with much worse accuracy.

In 1991 we started a research on another type of the explicitly correlated wave function-the Exponentially Correlated Gaussians (ECG). A full definition of the ECG wave function is as follows:

$$
\Psi=\hat{\mathcal{S}}\left(\Theta \sum_{k=1}^{K} c_{k} \psi_{k}\right),
$$

where $\hat{\mathcal{S}}$ is an operator ensuring proper electron and space symmetry of the function, and $\Theta$ is properly chosen spin function. The space part of the basis 
function is defined as

$$
\psi_{k}\left(\vec{r}_{1}, \ldots, \vec{r}_{n}\right)=\exp \left[-\sum_{i=1}^{n} a_{i, k}\left|\vec{r}_{i}-\vec{C}_{i, k}\right|^{2}-\sum_{i=1}^{n-1} \sum_{j=i+1}^{n} b_{i j, k}\left|\vec{r}_{i}-\vec{r}_{j}\right|^{2}\right],
$$

where $\mathrm{i}, j$ run over all the $n$ electrons, and $\mathrm{a}_{\mathrm{i}, \mathrm{k}}, \mathrm{b}_{\mathrm{ij}, \mathrm{k}}$ and $\vec{C}_{i, k}$ are nonlinear parameters determined variationally. As we can see the variables $\vec{r}_{i}$ occur in a quadratic form which is a feature of the Gaussian type functions. The first term of the exponent is common for one electron functions but the second one contains distances of all the pairs of electrons and is responsible for correlating the motion of the electrons. The elements mentioned above compose the name of the wave function-the Exponentially Correlated Gaussian function.

\section{The wave function optimization}

Every basis function $\psi_{k}$ contains, depending on the number of electrons, up to tens of nonlinear parameters and there are hundreds or thousands of such basis functions. Optimization of the total wave function, $\Psi$, requires a location of the minimum of the energy as a function of tens of thousands of parameters. To date there is no universal algorithm solving this type of problem and finding a global minimum for such a function is unfeasible. We have to accept the fact that a minimum obtained is merely a local one and put the effort on locating it as low as possible. It turns out that it brings satisfactory results.

In our algorithm we optimize (using Powell method [13]) simultaneously only $M$ parameters belonging to a single basis function with the other parameters kept fixed. When we determine the optimal subset of the parameters we move the optimization to the next basis function. After optimizing all the basis functions we close the cycle returning to the first one (see Fig. 2). Every step and every cycle lowers slightly the energy. Of course, the number of cycles is limited by an energy lowering criterion or, most frequently, by the cost of the computations.

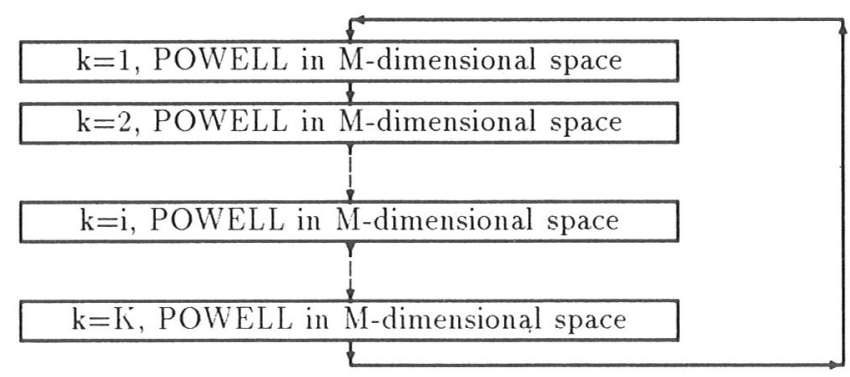

Figure 2: Optimization scheme. 
In the optimization process described above the updating and the diagonalization of the matrices is performed millions of times which makes the calculations very time consuming. Therefore it is very important to use an optimal code and machines. Thanks to the effort put into our algorithms we managed to diminish the computation time, from the pessimistic thousands of years predicted fewyears ago by Clementi [7, 12]. to days and weeks. Also the progress in computer technology allows not only to improve the quality of our results but also perform calculations unfeasible in the past.

The algebraic approximation (4) converts the Schrodinger equation (2) into the matrix form: $\mathcal{H C}=E \mathcal{S C}$. This is a common general symmetric eigenproblem which is solved in two basic stages:

- matrix elements calculation,

- matrix diagonalization.

The latter stage can be very effectively vectorized, especially when the operating system supplies with dedicated linear algebra libraries. Nowadays, a diagonalization of a dense $1000 \times 1000$ matrix makes neither time nor memory problems. During the optimization of a single basis function only one row and one column of the $\mathcal{H}$ and $\mathcal{S}$ matrices are updated. Utilization of this fact in our algorithm was one of the major steps leading to obtaining good results and performance.

The former stage, the matrices build-up, because of the mutual matrix elements independence, can be effectively parallelized. The loop running over the matrix indices comprises the standard object undergoing the parallelization. The following diagram (Fig. 3) presents a practical example of the speedup obtained when the procedure of filling up the matrices is well parallelized. This particular picture was obtained from the Atexpert performance monitor on the 16-processor Cray J916.

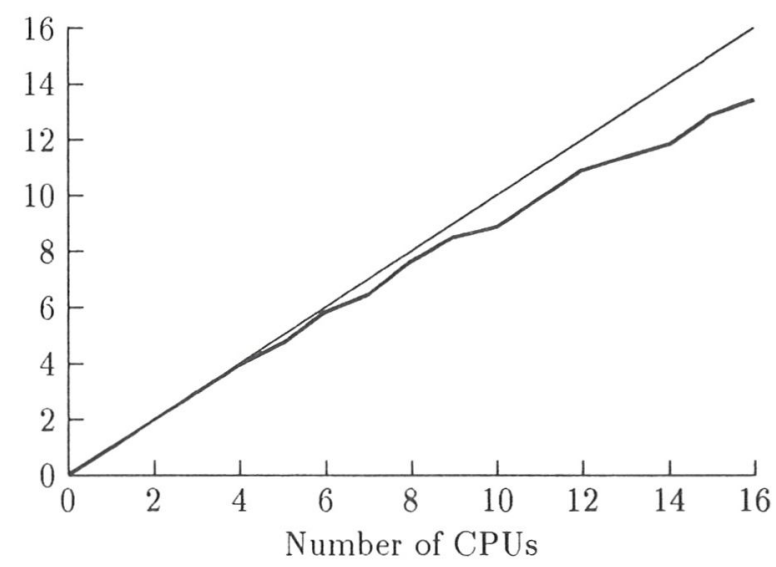

Figure 3: Performance speedup as a result of the parallelization. 
The access to the parallel computers is particularly important for extending our method to larger and larger systems-the cost of computing of one matrix element grows with the number of electrons as a factorial function. Regarding that, for fixed basis set size, the diagonalization cost remains constant, the effectiveness of the matrix elements computation stage becomes the most important factor of the whole approach. Massively parallel computers would be the most desired tool for this type of problem, especially when larger atoms and molecules are of interest. Figure 4 shows how dramatically grows the cost of the computations when going from two-electron to larger systems.

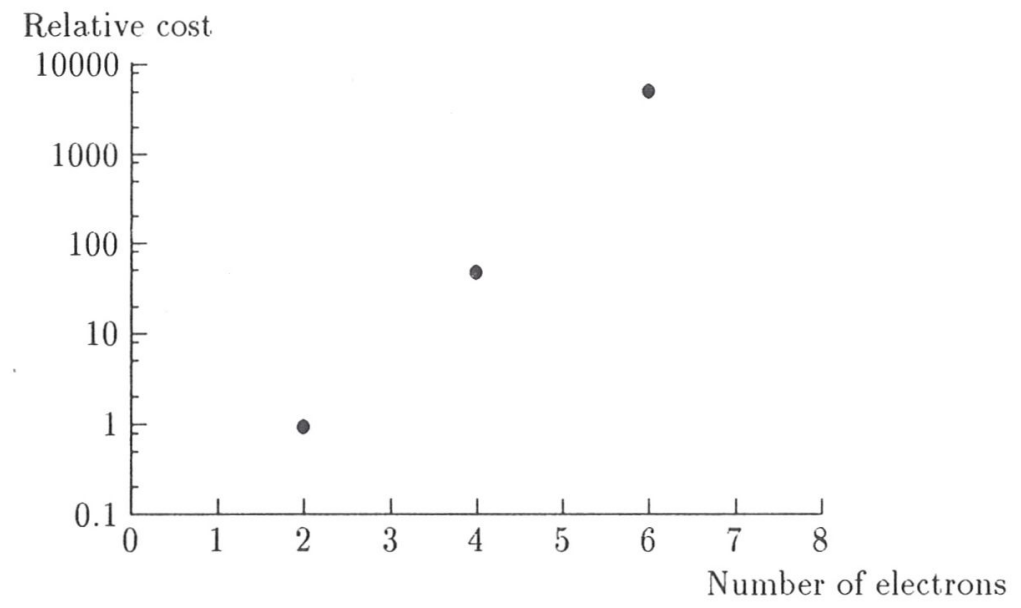

Figure 4: The cost of computation growth with the number of electrons.

\section{$6 \quad$ Results}

Results presented in this section summarize the last few years of our work on algorithms and source codes. The computer programs applied were created exclusively in our laboratory. The calculations were performed during last two years and some of the results were not published yet. All the computations were done at Adam Mickiewicz University or Poznań Supercomputing and Networking Center.

\subsection{2-electron systems}

For 2-electron molecules the ECG functions enable to solve the Schrödinger equation with an accuracy of at least 10 significant figures and, as we have shown on many examples [14, 15], are, from energetic point of view, equivalent to the Kołos-Wolniewicz wave functions. Table 2 collects the results for the hydrogen molecule in its ground and several excited states with various spin and space 
Table 2: 2-electron systems.

$\mathrm{H}_{2}$, ground state, $R=1.4011 \mathrm{bohr}$

\begin{tabular}{llc}
\hline & \multicolumn{1}{c}{ Energy $/ \mathrm{E}_{h}$} & Reference \\
\hline 1200-term ECG & -1.174475931211 & {$[16]$} \\
760 -term ECG & -1.1744759310 & this work \\
883-term KW & -1.1744759307 & {$[17]$} \\
full CI & -1.1743043 & {$[4]$} \\
\hline
\end{tabular}

$\mathrm{H}_{2}, a^{3} \Sigma_{g}^{+}$state, $R=1.8683$ bohr

600-term ECG $\quad-0.737156011$

177-term KW $\quad-0.737155958$

$\mathrm{H}_{2}, b^{3} \Sigma_{u}^{+}$state, $R=2.0$ bohr

600-term ECG $\quad-0.897076330 \quad[15]$

110 -term KW $\quad-0.897076017$

$\mathrm{H}_{2}, B^{1} \Sigma_{u}^{+}$state, $R=2.43$ bohr

\begin{tabular}{lll}
\hline 600 -term ECG & -0.756690483 & {$[15]$} \\
$\mathrm{KW}$ & -0.756690248 & {$[20]$} \\
\hline
\end{tabular}

$\mathrm{H}_{2}, C^{1} \Pi_{u}$ state, $R=1.952$ bohr

\begin{tabular}{lll}
\hline 600 -term ECG & -0.718368027 & {$[15]$} \\
$\mathrm{KW}$ & -0.718367979 & {$[21]$} \\
\hline
\end{tabular}

$\mathrm{H}_{2}, E F^{1} \Sigma_{g}^{+}$state, $R=1.5$ bohr

$\begin{array}{lll}600 \text {-term ECG } & -0.703000229 & {[15]} \\ \text { KW } & -0.702998510 & {[22]}\end{array}$

$\mathrm{H}_{3}^{+}$, ground state, $R_{12}=R_{13}=R_{23}=1.65 \mathrm{bohr}$

$\begin{array}{lll}600 \text {-term ECG } & -1.343835624 & {[15]} \\ \text { R12-CI } & -1.3438351 & {[23]}\end{array}$

$\mathrm{HeH}^{+}$, ground state, $R=1.46$ bohr

\begin{tabular}{lll}
\hline 600 -term ECG & -2.978706591 & {$[15]$} \\
$\mathrm{JC}$ & -2.97870262 & {$[24]$} \\
\hline
\end{tabular}


symmetries. In all the cases the energy obtained from the ECG is the most accurate ever published. The same quality of the results have been obtained for $\mathrm{HeH}^{+}$and $\mathrm{H}_{3}^{+}$. The choice of the systems presented in the tables proves that the ECG guarantee high quality of the results regardless of the spin or space symmetry, the charge or the number of nuclei. For the ground state of $\mathrm{H}_{2}$ the ECG functions yield the accuracy of a nanohartree. These are the most accurate calculations on a molecular system (except for the trivial 1-electron case) ever performed. In the case of the excited states we estimate the energy error as $0.001 \mathrm{c} \mathrm{m}^{-1}$ for the triplet $a$ and $b$ states and as $0.01 \mathrm{~cm}^{-1}$ for the singlet states: $B, C$ and EF. Similarly, for the $\mathrm{H}_{3}^{+}$the accuracy is about $0.001 \mathrm{~cm}^{-1}$, and for $\mathrm{HeH}^{+}$about $0.01 \mathrm{~cm}^{-1}$. In Table 2 our results are compared with the other best variational calculations. Obtaining results of this quality is presently possible on a personal computer in time of several hours.

\subsection{3-electron systems}

Among the 3-electron systems we have performed calculations for $\mathrm{H}_{3}$, helium dimer cation $\mathrm{He}_{2}^{+}$and for lithium atom (Table 3). The energies obtained are computed with the accuracy of 7 - 9 significant figures and, except for Li, are significantly more accurate then any other variational results. $\mathrm{H}_{3}$ is the molecule which Clementi group was struggled with. Contrary to their pessimistic predictions we can obtain an accuracy of $1 \mathrm{c} \mathrm{m}^{-1}$ in several days of computations on SGI R8000/75 type machine. For $\mathrm{He}_{2}^{+}$we estimate that the energy error is less then $1 \mathrm{~cm}^{-1}$. The latter calculations were performed for a wide range of the bond distance and were followed by rovibrational transition calculations. The computed transitions are on average $0.02 \mathrm{~cm}^{-1}$ in error when compared with spectroscopic data [25]. The calculations on $\mathrm{Li}$ atom are in progress. At present stage the energy error is ca. $0.001 \mathrm{~cm}^{-1}$.

\subsection{4-electron systems}

The accuracy of 7 digits is also attainable for 4-electron systems (Table 4) either atomic, $\mathrm{Be}$, or molecular, $\mathrm{He}_{2}$ and $\mathrm{LiH}$. In case of the beryllium atom the accuracy of our calculations is about $1 \mathrm{~cm}^{-1}$. The result has verified an experimental energy of beryllium. It turned out that the latter was slightly too high. Exceptionally accurate are the results for the helium dimer-the energy error is in the range of $0.05-0.09 \mathrm{~cm}^{-1}$. This is the first time when an analytical wave function yields such an accurate energy for a 4-electron system. At present, we are working 011 the whole energy curve which, hopefully, will contribute to the solution of the problem of vibrational stability of the system. The calculation on $\mathrm{LiH}$ is in progress. At present stage the estimated energy error is less than $9 \mathrm{c} \mathrm{m}^{-1}$. 
Table 3: 3-electron systems.

\begin{tabular}{llc}
\hline $\mathrm{H}_{3}$, ground state, $R_{1}=R_{2}=1.757$ bohr & \\
\hline & Energy $/ \mathrm{E}_{h}$ & Reference \\
\hline 600 -term ECG, 7200 par. & -1.65914905 & this work \\
Monte Carlo & -1.659156 & {$[26]$} \\
$\mathrm{CI}(1.7572)$ & -1.6589596 & {$[6]$} \\
\hline $\mathrm{He}+$, ground state, $R=2.042$ bohr & & \\
\hline 600 -term ECG, 5400 par. & -4.99464087 & this work \\
ICMRCI $(2.043)$ & -4.994284 & {$[5]$} \\
\hline Li, ground state & & this work \\
\hline 2172-term ECG, 13032 par. & -7.4780603175 & {$[27]$} \\
H-CI & -7.47806032310 & \\
\hline
\end{tabular}

Table 4: 4-electron systems.

\begin{tabular}{llc}
\hline Be, ground state & & \\
\hline & Energy $/ \mathrm{E}_{h}$ & Reference \\
\hline 1200-term ECG, 12000 par. & -14.66735502 & {$[28]$} \\
H-CI & -14.667350 & {$[29]$} \\
MCHF & -14.667113 & {$[8]$} \\
\hline
\end{tabular}

\begin{tabular}{llc}
$\mathrm{He}_{2}$, ground state, $R=5.6$ bohr & & \\
\hline 1600 -term ECG, 22400 par. & -5.807483462 & this work,[30] \\
Monte Carlo & -5.8074836 & {$[31]$} \\
full CI & -5.806110 & {$[9]$} \\
\hline
\end{tabular}

$\mathrm{LiH}$, ground state, $R=3.015$ bohr

600-term ECG, 8400 par. $\quad-8.070449 \quad$ this work

CCSDT1-R12 $-8.07048708 \quad$ [32]

\section{$7 \quad$ Summary}

The ECG functions have been known in quantum chemistry for over 30 years. The first results with these functions were published in 1960 [33, 34], However, accused of slow convergence, ECG were rejected for a long time. As we have proven recently this opinion was unjustified and today the ECG supply many best variational results. 
The application of the ECG functions has pointed out that the algebraic approximation can yield very good results. For the first time one can obtain a satisfactory convergence for systems with more than 2 electrons. This is a turning point in the computational quantum chemistry moving the research on a few-electron systems to a new level of development. Graphically the progress is represented by the new series of circles (ECG) on the following diagram.

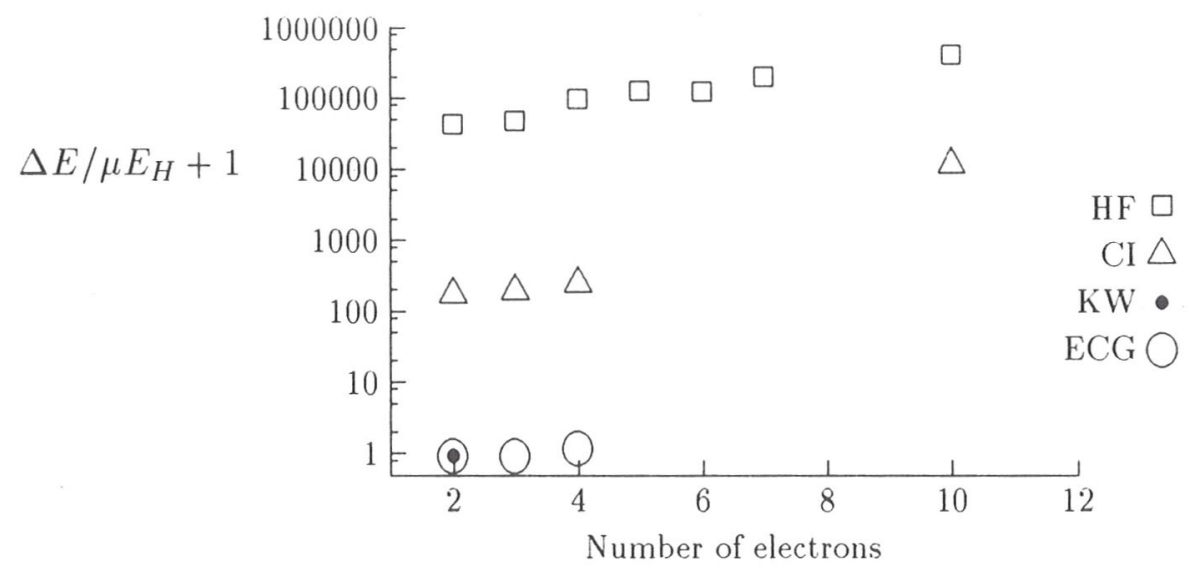

Figure 5: Total energy error, $\Delta \mathrm{E}$, obtained from $\operatorname{HF}(\cdot)$ and $\mathrm{CI}(\Delta)$ calculations and from explicitly correlated calculations with Kołos-Wolniewicz wave function $(\mathrm{KW}, \cdot)$. A new set of accurate results (ECG, $\bigcirc$ ) develops along the $\mathrm{X}$ axis.

\section{Acknowledgment}

This work was partially supported by the Polish Committee for Scientific Research (KBN 8 T11F 01008 p01 and KBN 2 P303 104 06). 


\section{References}

[1] H. M. James and A. S. Coolidge, J. Chem. Phys. 1, 825 (1933).

[2] W. Kołos and L. Wolniewicz, J. Chem. Phys. 41, 3663 (1964).

[3] W. Kołos and L. Wolniewicz, J. Chem. Phys. 49, 404 (1968).

[4] J. W. Liu and S. Hagström, Phys. Rev. A 48, 166 (1993).

[5] A. Carrington, C. H. Pyne, and P. J. Knowles, J. Chem. Phys. 102, 5979 (1995).

[6] K. P. Peterson. D. E. Woon, and T. H. Dunning Jr., J. Chem. Phys. 100, 7410 (1994).

[7] E. Clementi, G. C'orongiu, D. Bahattacharya. B. Feuston, D. Frve. A. Preiskorn, A. Rizzo, and W. Xue, Chem. Rev. 91. 679 (1991).

[8] C. Froese Fisher, J. Phys. B 26, 855 (1993).

[9] T. van Mourik and J. H. van Lenthe, J. Chem. Phys. 102, 7479 (1995).

[10] N. C. Handy, R. J. Harrison, P. J. Knowles, and H. F. Schaefer III, J. Phys. Chem. 88, 4852 (1984).

[11] E. A. Hylleraas, Z. Phys. 54, 347 (1929).

[12] E. Clementi, Int. J. Quantum Chem. 45, 511 (1993).

[13] M. J. D. Powell, Comput. J. 7, 155 (1964).

[14] J. Rychlewski, W. C'encek, and J. Komasa, Chem. Phys. Lett. 229, 657 (1994).

[15] W. Cencek, J. Komasa, and J. Rychlewski, Chem. Phys. Lett. 246, 417 (1995).

[16] W. Cencek and W. Kutzelnigg, J. Chem. Phys., submitted.

[17] L. Wolniewicz, J. Chem. Phys. 103, 1792 (1995).

[18] W. Kołos and J. Rychlewski, J. Mol. Spectr. 169, 341 (1995).

[19] W. Kołos and J. Rychlewski, J. Mol. Spectr. 143, 237 (1990).

[20] L. Wolniewicz, private communication.

[21] L. Wolniewicz. Chem. Phys. Lett. 233, 644 (1995). 
[22] L. Wolniewicz and K. Dressier, J. Chem. Phys. 100, 444 (1994).

[23] R. Röhse, W. Klopper, and W. Kutzelnigg, J. Chem. Phys. 99, 8830 (1993).

[24] D. M. Bishop and L. M. Cheung, J. Mol. Spectr. 75, 462 (1979).

[25] W. Cencek and J. Rychlewski, J. Chem. Phys. 102, 2533 (1995).

[26] D. L. Diedrich and J. B. Anderson, J. Chem. Phys. 100, 8089 (1994).

[27] Z-C. Yan and G. W. F. Drake, Phys. Rev. A 52, R4316 (1995).

[28] J. Komasa, W. Cencek, and J. Rychlewski, Phys. Rev. A 52, 4500 (1995).

[29] G. Büsse and H. Kleindienst, Phys. Rev. A 51, -5019 (1995).

[30] J. Komasa and J. Rychlewski, Chem. Phys. Lett. 249. 253 (1996).

[31] B. Chen and J. B. Anderson. J. Chem. Phys. 102, 2802 (1995).

[32] J. Noga, D. Tunega, W. Klopper. and W. Kutzelnigg, J. Chem. Phys. 103, 309 (1995).

[33] S. F. Boys, Proc. Roy. Soc. A 258. 402 (1960).

[34] K. Singer, Proc. Roy. Soc. A 258. 412 (1960). 